\title{
Effect of a mobile application and smart devices on heart rate variability in diabetic patients with high cardiovascular risk: A sub-study of the LIGHT randomized clinical trial
}

\author{
Mert IIlker Hayıroğlu', Göksel Çinier', Gizem Yüksel', Levent Pay', Furkan Durak', Tufan Çınar², Duygu İnan',

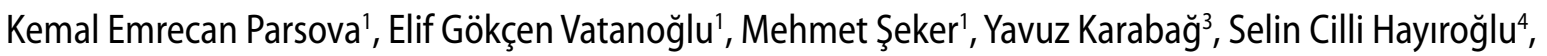 \\ Can Altundaş', Ahmet İlker Tekkeşin' \\ 'Department of Cardiology, Dr. Siyami Ersek Thoracic and Cardiovascular Surgery Training and Research Hospital, Istanbul, Turkey \\ 2Department of Cardiology, Haydarpasa Sultan II Abdulhamid Han Training and Research Hospital, Istanbul, Turkey \\ ${ }^{3}$ Department of Cardiology, Kafkas University Faculty of Medicine, Istanbul, Turkey \\ ${ }^{4}$ Department of Rheumatology, Marmara University Faculty of Medicine, Istanbul, Turkey
}

Correspondence to: Mert İlker Hayıroğlu, MD, Department of Cardiology, Dr. Siyami Ersek Thoracic and Cardiovascular Surgery Training and Research Hospital, 34768 Istanbul, Turkey phone: +90216715 4089 e-mail:mertilkerh@yahoo.com Copyright by the Author(s), 2021 Kardiol Pol. 2021; 79 (11): 1239-1244; DOI: 10.33963/KP.a2021.0112

Received:

May 7, 2021

Revision accepted: September 20, 2021

Published online: September 20, 2021

\begin{abstract}
A B S TRA C T
Background: This investigation aims to evaluate the effect of a mobile application and smart devices on frequency and time domains of heart rate variability (HRV) in diabetic patients in 1-year follow-up. Methods: This is post-hoc analysis of a diabetic subgroup of "Lifestyle Intervention usinG mobile technology in patients with high cardiovascular risk: a pragmatic randomized clinical Trial" (LIGHT). One hundred and nine and 118 patients were enrolled in two arms: the intervention plus usual care and the usual care arm. The study outcome was the 1-year HRV parameters adjusted to the baseline HRV parameters. HRV measures were recorded for every patient at the randomization and final visits with 24-hour Holter monitoring.
\end{abstract}

Results: There was an improvement in the standard deviation of normal to normal (SDNN) R-R intervals 24 -hour by 4.8 (adjusted treatment effect $4.8,95 \%$ confidence interval [Cl], 0.1-9.5; $P=0.044$ ) in the intervention-plus-usual-care arm compared to usual care after a 1-year follow-up. The improvement was also experienced in other HRV time domains including standard deviation of the mean R-R intervals calculated over a 5-minute period, SDNN, square root of the mean squared difference of successive $R-R$ intervals, and the percentage of the differences between adjacent normal R-R intervals exceeding 50 milliseconds. A significant enhancement was also detected in HRV frequency domains of total power low frequency and high frequency in the intervention plus usual care compared to usual care after a 1-year follow-up.

Conclusions: The mobile application and smart device technology compared to usual care alone improved HRV parameters in diabetic patients at 1-year follow-up.

Key words: diabetes mellitus, heart rate variability, mobile application

Kardiol Pol 2021; 79, 11: 1239-1244

\section{INTRODUCTION}

The autonomic nervous system (ANS) degenerates with aging resulting in increased sympathetic and diminished parasympathetic activity [1]. In addition to aging, chronic diseases, including diabetes mellitus and atherosclerotic cardiovascular disease, seriously influence ANS and deteriorate parasympathetic cardio-protection $[2,3]$. There are numerous methods evaluating the function of ANS. Heart rate variability (HRV), which is defined as the indirect calculation for ANS function, is a well-accepted prognostic measure in several diseases such as brain injury and heart failure $[4,5]$. ANS dysfunction frequently occurs in diabetic patients due to impairment of 
WHAT'S NEW?

Mobile technology-based solutions have promising effects in the management of chronic diseases such as diabetes mellitus. Diabetes mellitus causes autonomic nervous system dysfunction, secondary to its degenerative effect on the nervous system in long-term follow-up. However, improvement in heart rate variability might be possible with an approach including technology-based systems in high cardiovascular risk patients without evidence of diabetic vascular complications. In our study, a mobile application and smart device technology compared to usual care alone improved both frequency and time domains of heart rate variability in diabetic patients at 1-year follow-up.

autonomic fibers innervating blood vessels and the heart [6]. Decreased HRV has been linked to cardiac autonomic neuropathy and mortality in a long-term follow-up of diabetes [7]. Moreover, although patients have asymptomatic diabetes, HRV is reported to be a predictor of major adverse cardiac events $[8,9]$.

Mobile technology-based solutions have promising effects in the management of chronic diseases. Diabetes mellitus, which is the most important example of chronic disease, has compelling management for both physicians and patients. A standard approach to diabetes should lead patients to a healthy lifestyle in addition to individualized medications. Mobile health systems offer a multi-faceted method in diabetes treatment and follow-up providing indispensable contacts with patients, which facilitate providing information about healthy lifestyles and enable accurate glycemic control $[10,11]$. However, despite the rapidly expanding field of mobile systems in diabetes, there is a lack of evidence for the effect of mobile applications on the HRV parameters in diabetic patients with high cardiovascular risk in a long-term follow-up. Therefore, in the current study, we aim to evaluate the effect of lifestyle intervention using mobile technology and smart devices on frequency and time domains of HRV in diabetic patients in a 1-year follow-up.

\section{METHODS}

This investigation was a sub-study of lifestyle intervention using mobile technology in patients with high cardiovascular risk: A pragmatic randomized clinical Trial (LIGHT) implemented in the tertiary cardiovascular center from February 2018 to March 2020 [12]. In the LIGHT trial, the participants, who were between 20 and 79 years of age and at high risk for cardiovascular diseases, as indicated by a 10-year atherosclerotic cardiovascular disease (ASCVD) risk score of $\geq 7.5 \%$, were randomly allocated to either arm: the intervention plus usual care or usual care (NCT03397849). The design of the LIGHT was published previously [13]. All of the diabetic patients $(n=227)$ of LIGHT $(n=483)$ were included as the study population in this investigation. One hundred and eighteen diabetic patients from the usual care arm and 109 from the intervention-plus-usual-care arm constituted the study population. All the patients received standard usual care which is routinely applied to diabetic patients in our center in accordance with the guidelines. Patients in the intervention-plus-usual-care arm received training on our specially designed mobile application and smart devices, which were distributed to the patients in compliance with the study design [13]. The daily blood pressure, step count, diet, and weight sections were monitored through 1 year, and pre-specified motivational messages were delivered to every patient intending to lead a healthy lifestyle. Both the intervention-plus-usual-care and usual care groups were evaluated with 24-hour rhythm Holter monitoring at the beginning and final visits. The baseline characteristics, laboratory parameters, and echocardiographic features were compared between these groups. The participants provided written informed consent at the beginning of this prospective study.

Twenty-four-hour rhythm Holter monitoring was performed twice for each study participant at the beginning and final visits. Numerous indices of time and frequency domains were used to measure HRV. The following parameters were retrieved from the Holter recordings: standard deviation of normal to normal (SDNN) R-R intervals 24-hour, standard deviation of the mean R-R intervals calculated over a 5-minute period (SDANN index), mean of the standard deviation of R-R intervals calculated over a 5-minute period (SDNN index), square root of the mean squared difference of successive R-R intervals ( $r M S S D$ ), and the percentage of the differences between adjacent normal $\mathrm{R}-\mathrm{R}$ intervals exceeding $50 \mathrm{~ms}$ (pNN50). In the frequency domains, the variance of all normal-to-normal intervals approximately representing total power (TP), high frequency (HF) power representing parasympathetic activity, low frequency (LF) power representing both sympathetic and parasympathetic activity, and LF/HF ratio representing the sympathovagal balance were analyzed.

\section{Statistical analysis}

Analyses were performed using Statistical Package for Social Sciences software, version 20.0 (SPSS; IBM, Armonk, NY, USA). The normality (homogeneity) of the variables was evaluated using the Kolmogorov-Smirnov test with Lilliefors correction and equality of variance was tested using the Levene test. The study group was divided into two arms: the usual care arm and the and intervention plus usual care arm according to their randomization in the LIGHT trial. Baseline characteristics, laboratory parameters, echocardiographic variables, and medications were compared between these groups. Data were presented as mean (standard deviation [SD]) for normally 
distributed data, and as median (interquartile range) for continuous variables that were not normally distributed. Continuous variables were analyzed by independent t-test if normally distributed. The Mann-Whitney U test was applied for comparisons of the data that were not normally distributed. Categorical data were presented as $\mathrm{n}(\%)$ and analyzed by using chi-square or Fisher's exact tests. We used $x 2$ tests to compare separately baseline and 1-year follow-up medication intake. Repeated measurement of analysis of covariance (ANCOVA) was used to evaluate the outcomes. The frequency and time domains of HRV were each examined with a 2-by-2 simplified model of ANCOVA intervention plus usual care versus usual care as the between subject factor, time (baseline, 1-year follow-up) as the within-subjects factor, and adjusted for baseline $\mathrm{HbA1c}$, 1-year HbA1c, baseline and 1-year anti-hypertensive and beta-blocker data for evaluating between-groups differences. For each ANCOVA, the main hypothesis of interest was the 2-way (group by time) with a Bonferroni-corrected alpha of 0.025 (2 time points). To enable comparison of between-group effect sizes, standardized mean score differences (SMDs) were calculated by dividing mean score differences between groups by the pooled standard deviation. The results were presented as the adjusted mean difference (95\% confidence interval $[\mathrm{Cl}]$ ).

\section{RESULTS}

Baseline and 1-year demographic characteristics, laboratory variables, and echocardiographic parameters were listed in Table 1. This sub-study of the LIGHT trial included a total of 227 patients (mean age 57.1 [7.4] years; $47.1 \%$ male). The groups did not differ in terms of age, sex, body mass index, and blood pressure measurements. Left ventricular ejection fraction, left atrial volume index, and left ventricular mass index were similar between the usual care and intervention plus usual care groups. Considering laboratory parameters, $\mathrm{HbA}_{1 \mathrm{c}}$ and fasting blood glucose were notably higher in the intervention-plus-usual-care group compared to the usual care group $(P=0.008$ and $P<0.001$ respectively). Table 2 presented the medications in two groups at baseline and 1-year. The usual care group and the intervention-plus-usual-care group were similar in terms of oral antidiabetic drugs and insulin usage.

Table 1. Baseline characteristics

\begin{tabular}{|c|c|c|c|}
\hline & Usual care $(n=118)$ & Intervention plus usual care $(n=109)$ & P-value \\
\hline Age, years & $57.2(7.6)$ & $57.1(7.2)$ & 0.89 \\
\hline Gender, male \% & $55(46.6)$ & $52(47.7)$ & 0.86 \\
\hline Body mass index, $\mathrm{kg} / \mathrm{m}^{2}$ & $30.0(26.7-33.3)$ & $30.2(27.4-34.0)$ & 0.62 \\
\hline Systolic blood pressure, $\mathrm{mm} \mathrm{Hg}$ & $143(15)$ & $140(18)$ & 0.19 \\
\hline Diastolic blood pressure, $\mathrm{mm} \mathrm{Hg}$ & $84(13)$ & $83(15)$ & 0.74 \\
\hline Hypertension, \% & $84(71.2)$ & $83(76.1)$ & 0.28 \\
\hline Hyperlipidemia, \% & $32(27.1)$ & $35(32.1)$ & 0.72 \\
\hline Smoking, \% & $35(29.7)$ & $43(39.4)$ & 0.12 \\
\hline Heart rate, beat per minute & $75(5)$ & $71(6)$ & 0.52 \\
\hline Left ventricular ejection fraction, $\%$ & $61(2)$ & $61(2)$ & 0.81 \\
\hline Left atrial volume index, $\mathrm{ml} / \mathrm{m}^{2}$ & $28.5(7.8)$ & $29.9(8.4)$ & 0.14 \\
\hline Left ventricular mass index, $\mathrm{g} / \mathrm{m}^{2}$ & $83.9(19.3)$ & $81.6(17.2)$ & 0.46 \\
\hline Creatinine, mg/dl & $0.85(0.15)$ & $0.85(0.16)$ & 0.95 \\
\hline Baseline LDL-cholesterol, mg/dl & $132(113-159)$ & $129(106-155)$ & 0.35 \\
\hline 1-year LDL-cholesterol, mg/dl & $114(95-140)$ & $111(91-123)$ & 0.031 \\
\hline Baseline HDL-cholesterol, mg/dl & $42(37-49)$ & $42(37-49)$ & 0.96 \\
\hline 1-year HDL-cholesterol, mg/dl & $42(36-48)$ & $44(40-50)$ & 0.043 \\
\hline Baseline triglyceride, mg/dl & $161(125-225)$ & $168(122-215)$ & 0.97 \\
\hline 1-year triglyceride, mg/dl & $176(135-226)$ & $131(95-175)$ & $<0.001$ \\
\hline $\mathrm{Hs}-\mathrm{CRP}, \mathrm{mg} / \mathrm{dl}$ & $0.20(0.00-0.40)$ & $0.00(0.00-0.30)$ & 0.07 \\
\hline Hs-troponin, ng/ml & $0.002(0.001-0.003)$ & $0.002(0.001-0.003)$ & 0.29 \\
\hline Pro-BNP, pg/ml & $33.5(13.4-43.1)$ & $27.0(12.0-55.0)$ & 0.84 \\
\hline Baseline HbA1c, \% & $7.4(1.5)$ & $7.0(1.3)$ & 0.008 \\
\hline 1-year HbA1c, \% & $7.1(1.4)$ & $6.7(1.0)$ & 0.002 \\
\hline Baseline glucose, mg/dl & $109(97-132)$ & $130(109-157)$ & $<0.001$ \\
\hline 1-year glucose, mg/dl & $123(107-147)$ & $105(100-123)$ & $<0.001$ \\
\hline $\mathrm{TSH}, \mathrm{mlU} / \mathrm{l}$ & $1.6(1.0-2.2)$ & $1.5(1.0-2.2)$ & 0.98 \\
\hline $\mathrm{T} 4, \mathrm{ng} / \mathrm{dl}$ & $1.0(0.9-1.0)$ & $1.0(0.9-1.1)$ & 0.14 \\
\hline Baseline ASCVD score & $16.3(11.1-24.2)$ & $17.0(11.9-26.4)$ & 0.46 \\
\hline 1-year ASCVD score & $18.2(11.1-26.8)$ & $14.7(10.6-23.2)$ & 0.027 \\
\hline
\end{tabular}

Continuous variables are presented as mean standard deviation [SD] for normally distributed data, and as median (interquartile range [IQR]) for that are not normally distributed Data are presented as number (percentage) of patients

Abbreviations: ASCVD, atherosclerotic cardiovascular disease risk score; BMI, body mass index; BNP, brain natriuretic peptide; DBP, diastolic blood pressure; HDL, high-density lipoprotein; hs-CRP, high-sensitive C-reactive protein; hs-troponin, high-sensitive troponin; HT, hypertension; LDL, low-density lipoprotein; LVEF, left ventricular ejection fraction; SBP, systolic blood pressure 
Table 2. The medication status of baseline and 1-year visit of the usual care arm and the intervention plus usual care arm

\begin{tabular}{|c|c|c|c|c|}
\hline & \multicolumn{2}{|c|}{ Usual care $(n=118)$} & \multicolumn{2}{|c|}{ Intervention plus usual care $(n=109)$} \\
\hline & Baseline & At 1 year & Baseline & At 1 year \\
\hline \multirow[t]{2}{*}{ Antiplatelet drugs } & 19.5 & 19.5 & 19.3 & 19.3 \\
\hline & \multicolumn{4}{|c|}{$P^{\mathrm{a}}=0.96 ; P^{\mathrm{b}}=0.96$} \\
\hline \multirow[t]{2}{*}{ Statin } & 4.2 & 76.3 & 8.3 & 76.1 \\
\hline & \multicolumn{4}{|c|}{$P^{a}=0.20 ; P^{b}=0.98$} \\
\hline \multirow[t]{2}{*}{ ACE inhibitors/ARB } & 53.4 & 55.1 & 63.3 & 65.1 \\
\hline & \multicolumn{4}{|c|}{$P^{\mathrm{a}}=0.13 ; P^{\mathrm{b}}=0.12$} \\
\hline \multirow[t]{2}{*}{ Calcium channel inhibitors } & 27.1 & 30.5 & 27.5 & 39.4 \\
\hline & \multicolumn{4}{|c|}{$P^{\mathrm{a}}=0.94 ; P^{\mathrm{b}}=0.15$} \\
\hline \multirow[t]{2}{*}{ Beta blockers } & 25.4 & 26.3 & 21.1 & 29.4 \\
\hline & \multicolumn{4}{|c|}{$P^{\mathrm{a}}=0.44 ; P^{\mathrm{b}}=0.60$} \\
\hline \multirow[t]{2}{*}{ Diuretics } & 8.5 & 9.3 & 8.3 & 11.9 \\
\hline & \multicolumn{4}{|c|}{$P^{\mathrm{a}}=0.95 ; P^{\mathrm{b}}=0.52$} \\
\hline \multirow[t]{2}{*}{ Oral antidiabetic drugs } & 84.7 & 93.2 & 86.2 & 94.5 \\
\hline & \multicolumn{4}{|c|}{$P^{a}=0.75 ; P^{b}=0.69$} \\
\hline \multirow[t]{2}{*}{ Insulin } & 15.2 & 16.9 & 15.6 & 16.5 \\
\hline & \multicolumn{4}{|c|}{$P^{\mathrm{a}}=0.94 ; P^{b}=0.93$} \\
\hline
\end{tabular}

ap value presents the comparison of the baseline variables; ${ }^{b} P$ value presents the comparison of 1 -year variables

Data are presented as number (percentage) of patients

Abbreviations: $A C E$, angiotensin-converting enzyme; $A R B$, angiotensin receptor blockers

Table 3. Endpoint analyses in the usual care and the intervention plus usual care arms

\begin{tabular}{|c|c|c|c|c|c|c|}
\hline & \multicolumn{2}{|c|}{ Usual care $(n=118)$} & \multicolumn{2}{|c|}{$\begin{array}{l}\text { Intervention plus usual care } \\
\qquad(\mathrm{n}=109)\end{array}$} & \multirow{2}{*}{$\begin{array}{l}\text { Estimated } \\
\text { treatment effect } \\
\text { (mean differencea } \\
\text { with } 95 \% \mathrm{CI} \text { ) }\end{array}$} & \multirow[t]{2}{*}{$P$-value } \\
\hline & Baseline & At 1 year & Baseline & At 1 year & & \\
\hline \multicolumn{7}{|c|}{ Study outcomes: HRV time domains } \\
\hline SDNN $24 \mathrm{~h}, \mathrm{~ms}$ & $159(17)$ & $154(18)$ & $150(16)$ & $153(18)$ & $4.85(0.12-9.57)$ & 0.044 \\
\hline SDANN index, ms & $147(23)$ & $140(21)$ & $133(20)$ & $144(19)$ & $5.66(0.33-10.98)$ & 0.037 \\
\hline SDNN index, ms & $69(20)$ & $65(19)$ & $57(17)$ & $61(17)$ & $8.33(3.37-13.28)$ & 0.001 \\
\hline RMSSD, ms & $32(13)$ & $31(15)$ & $36(7)$ & $40(7)$ & $-7.27(-10.26-[-4.28])$ & $<0.001$ \\
\hline PNN50, \% & $20(9)$ & $20(9)$ & $20(9)$ & $25(9)$ & $-2.42(-4.97-0.12)$ & 0.06 \\
\hline \multicolumn{7}{|c|}{ HRV frequency domains } \\
\hline $\mathrm{TP}, \mathrm{msec}^{2}$ & $2304(515)$ & $2198(525)$ & $2442(499)$ & 2745 (519) & $-349.06(-482.92-[-215.19])$ & $<0.001$ \\
\hline $\mathrm{LF}, \mathrm{msec}^{2}$ & $558(139)$ & $539(140)$ & $586(153)$ & $634(157)$ & $-57.74(-96.90-[-18.59])$ & 0.004 \\
\hline $\mathrm{HF}, \mathrm{msec}^{2}$ & $204(63)$ & $201(63)$ & $246(61)$ & $261(61)$ & $-52.10(-68.65-[-35.55])$ & $<0.001$ \\
\hline LF/HF & $2.94(0.95)$ & $2.87(0.88)$ & $2.43(0.52)$ & $2.48(0.51)$ & $0.48(0.29-0.68)$ & $<0.001$ \\
\hline
\end{tabular}

adjusted for baseline $\mathrm{HbA}_{1 c^{\prime}} 1$-year $\mathrm{HbA}_{1 c^{\prime}}$ baseline and 1-year anti-hypertensive and $\beta$-blocker

Data were presented as mean (standard deviation [SD]) for normally distributed continuous variables

Abbreviations: $H F$, high frequency; HRV, heart rate variability; LF, low frequency; pNN50, percentage of the differences between adjacent normal R-R intervals exceeding 50 milliseconds; RMSSD, square root of the mean squared difference of successive R-R intervals; SDANN index, standard deviation of the mean R-R intervals calculated over a 5-minute period; SDNN, standard deviation of normal to normal R-R intervals; SDNN index, mean of the standard deviation of R-R intervals calculated over a 5-minute period; TP, total power

In the intervention-plus-usual-care arm, the baseline SDNN 24-hours was 150 (16) and the 1-year SDNN 24-hours was 153 (18). In the usual care arm, the corresponding values were 159 (17) and 154 (18), respectively. The estimated treatment effect of intervention plus usual care over usual care on the 1-year SDNN 24-hours according to regression modeling was $4.8 \%$ (adjusted treatment effect $4.8,95 \% \mathrm{Cl}$, 0.1-9.5; $P=0.044$ ) (Table 3). Other changes of HRV time domains were listed in Table 3 grouped according to baseline and 1-year results in the usual care and intervention plus usual care arms. The estimated treatment effect on other HRV time domains was all in favor of the intervention-plus-usual-care group compared to the usual care group. In the intervention-plus-usual-care arm, the baseline
TP was 2442 (499) and the 1-year TP was 2745 (519). In the usual care arm, the corresponding values were 2304 (515) and 2198 (525), respectively. The estimated treatment effect on other HRV frequency domains was all in favor of the intervention-plus-usual-care group compared to the usual care group.

\section{DISCUSSION}

In a 1-year follow-up, intervention with the mobile application and smart devices significantly improved both time and frequency domains of HRV compared to usual care alone. Our study appears as an indicator of the modifiable structure of HRV with a healthy lifestyle in diabetic patients. Although diabetes mellitus results 
in ANS dysfunction secondary to the natural course of the disease in a long-term follow-up, improvement in HRV might be possible with technology-based systems in high cardiovascular risk patients without evidence of diabetic vascular complications. Following the results of this sub-group analysis of the LIGHT trial, the mobile application and smart device technology demonstrated to have positive effects on prognostic HRV parameters for the first time in the literature. There was no difference between women and men in maintaining an adequate lifestyle throughout the investigation. Since cardiovascular disease prevention plays an important role in women, there was no difference in the study population in terms of adherence to lifestyle. Moreover, the daily mean step count of the patients did not have a relation with the age of the patients.

Mobile application systems have been found both beneficial and useful in previous trials regarding heart failure, atrial fibrillation, and primary prevention of cardiovascular diseases $[12,14,15]$. Moreover, these systems have presented trustworthy positive results in the self-management of diabetes mellitus [16]. The adhibition of mobile applications such as the Alive-PD (NutritionQuest) and the Mobile Diabetes Prevention Program (mDPP) has demonstrated a positive effect on promoting lifestyle modifications in diabetic patients $[17,18]$. Significant weight reduction has been also achieved with a mobile application in diabetic patients in a 3-month period [19]. However, there is a lack of data about the effect of mobile applications on well-accepted study endpoints in diabetic patients. Thus, our study bridges the gap in the literature regarding the effect of the technology-based systems on ANS. Improvement in HRV parameters might be possible with the aid of a well-structured multifaceted application and follow-up system similar to ours.

Our mobile application system included healthy lifestyle guidance including physical exercise and dietary recommendations. It has been reported that weight loss using lifestyle interventions has a positive impact on sympathetic and parasympathetic HRV indices of cardiac autonomic function [20]. Furthermore, physical exercise has been proved to improve HRV in patients with diabetes [21]. Although HRV is not an obligatory measurement in the management of diabetes, it has a predictive and prognostic value owing to its association with diabetic complications and mortality [22]. Inappropriate management of diabetes results both in increased $\mathrm{HbA} 1 \mathrm{c}$ levels and hypoglycemic attacks, which is linked to reduced HRV levels [23]. Therefore, a change in HRV parameters appears as a an important endpoint in diabetic patients. Moreover, since diabetes causes ANS dysfunction, it is difficult to improve HRV parameters in diabetic patients. For this reason, this investigation exhibits evidence supporting the effectiveness of mobile health-based systems in diabetic patients with high cardiovascular risk.

\section{Study limitations}

Our investigation has several limitations due to its design and implementation. First, the patients were recruited and followed up in a single center. Second, the data entry was not a hundred percent, but the compliance increased with the aid of automatic messages and individualized phone calls inviting patients for an outpatient visit. Eighty-three percent of data input frequency was accomplished using the methods in the study design; however, it may not represent the 1-year follow-up due to missing data. Third, the study was implemented with individually provided data; however, if data were not compatible, they were verified through phone calls and invitations to outpatient visits. Finally, a 1-year follow-up might limit the results of this study because it might be a short duration to evaluate the changes on HRV.

\section{CONCLUSION}

The mobile application and smart device technology compared to usual care alone improved both frequency and time domains of HRV in diabetic patients at a 1-year follow-up.

\section{Article information}

Funding: This work was supported by TürkTelekomunikasyon A.Ş. The study was performed at Dr. Siyami Ersek Thoracic and Cardiovascular Surgery Training and Research Hospital, Turkey.

Conflict of interest: None declared.

Open access: This article is available in open access under Creative Common Attribution-Non-Commercial-No Derivatives 4.0 International (CC BY-NC-ND 4.0) license, allowing to download articles and share them with others as long as they credit the authors and the publisher, but without permission to change them in any way or use them commercially. For commercial use, please contact the journal office at kardiologiapolska@ptkardio.pl.

How to cite: Hayıroğlu MI, Çinier G, Yüksel G, et al. Effect of a mobile application and smart devices on heart rate variability in diabetic patients with high cardiovascular risk: a sub-study of the LIGHT randomized clinical trial. Kardiol Pol. 2021; 79(11): 1239-1244, doi: 10.33963/KP.a2021.0112.

\section{REFERENCES}

1. Goswami N, Abulafia C, Vigo D, et al. Falls risk, circadian rhythms, and melatonin: current perspectives. Clin Interv Aging. 2020; 15: 2165-2174, doi: 10.2147/CIA.S283342, indexed in Pubmed: 33204081.

2. Agashe $S$, Petak S. Cardiac autonomic neuropathy in diabetes mellitus. Methodist Debakey Cardiovasc J. 2018; 14(4): 251-256, doi: 10.14797/mdcj-14-4-251, indexed in Pubmed: 30788010.

3. Wichterle D, Simek J, La Rovere MT, et al. Prevalent low-frequency oscillation of heart rate: novel predictor of mortality after myocardial infarction. Circulation. 2004; 110(10): 1183-1190, doi: 10.1161/01. CIR.0000140765.71014.1C, indexed in Pubmed: 15313954.

4. Lu HY, Huang APH, Kuo LT. Prognostic value of variables derived from heart rate variability in patients with traumatic brain injury after decompressive surgery. PLoS One. 2021; 16(2): e0245792, doi: 10.1371/journal. pone.0245792, indexed in Pubmed: 33539419

5. Huikuri HV, Stein PK. Heart rate variability in risk stratification of cardiac patients. Prog Cardiovasc Dis. 2013; 56(2): 153-159, doi: 10.1016/j. pcad.2013.07.003, indexed in Pubmed: 24215747.

6. Valensi P, Extramiana F, Lange C, et al. DESIR Study Group. Influence of blood glucose on heart rate and cardiac autonomic function. The 
DESIR study. Diabet Med. 2011; 28(4): 440-449, doi: 10.1111/j.14645491.2010.03222.x, indexed in Pubmed: 21204961.

7. Maser RE, Mitchell BD, Vinik Al, et al. The association between cardiovascular autonomic neuropathy and mortality in individuals with diabetes: a meta-analysis. Diabetes Care. 2003; 26(6): 1895-1901, doi: 10.2337/diacare.26.6.1895, indexed in Pubmed: 12766130.

8. Valensi P, Sachs RN, Harfouche B, et al. Predictive value of cardiac autonomic neuropathy in diabetic patients with or without silent myocardial ischemia. Diabetes Care. 2001; 24(2): 339-343, doi: 10.2337/diacare.24.2.339, indexed in Pubmed: 11213889.

9. Plaza-Florido A, Sacha J, Alcantara J. Short-term heart rate variability in resting conditions: methodological considerations. Kardiol Pol. 2021;79(78): 745-755, doi: 10.33963/KP.a2021.0054, indexed in Pubmed: 34227676.

10. Liang X, Wang $Q$, Yang X, et al. Effect of mobile phone intervention for diabetes on glycaemic control: a meta-analysis. Diabet Med. 2011;28(4):455-463, doi: 10.1111/j.1464-5491.2010.03180.x, indexed in Pubmed: 21392066.

11. Bonoto BC, de Araújo VE, Godói IP, et al. Efficacy of mobile apps to support the care of patients with diabetes mellitus: a systematic review and meta-analysis of randomized controlled trials. JMIR Mhealth Uhealth. 2017; 5(3): e4, doi: 10.2196/mhealth.6309, indexed in Pubmed: 28249834.

12. Tekkeşin Al, Hayıroğlu Mi, Çinier G, et al. Lifestyle intervention using mobile technology and smart devices in patients with high cardiovascular risk: A pragmatic randomised clinical trial. Atherosclerosis. 2021; 319:21-27, doi: 10.1016/j.atherosclerosis.2020.12.020, indexed in Pubmed: 33465658.

13. Tekkesin I, Cinier G, Hayiroglu M. Rationale and design of lifestyle intervention using mobile technology in patients with high cardiovascular risk: a pragmatic randomized clinical trial. Turk Kardiyol Dern Ars. 2020; 48(2): 149-157, doi: 10.5543/tkda.2019.69494, indexed in Pubmed: 32147654.

14. Guo X, Gu X, Jiang J, et al. A hospital-community-family-based telehealth program for patients with chronic heart failure: single-arm, prospective feasibility study. JMIR Mhealth Uhealth. 2019; 7(12): e13229, doi: 10.2196/13229, indexed in Pubmed: 31833835.

15. Guo Y, Lane DA, Wang L. Mobile health technology to improve care for patients with atrial fibrillation. J Am Coll Cardiol. 2020; 75(13): 1523-1534, doi: 10.1016/j.jacc.2020.01.052, indexed in Pubmed: 32241367.
16. Quinn CC, Shardell MD, Terrin ML, et al. Cluster-randomized trial of a mobile phone personalized behavioral intervention for blood glucose control. Diabetes Care. 2011; 34(9): 1934-1942, doi: 10.2337/dc11-0366, indexed in Pubmed: 21788632.

17. Block G, Azar KMj, Romanelli RJ, et al. Diabetes prevention and weight loss with a fully automated behavioral intervention by email, web, and mobile phone: a randomized controlled trial among persons with prediabetes. J Med Internet Res. 2015; 17(10): e240, doi: 10.2196/jmir.4897, indexed in Pubmed: 26499966.

18. Fukuoka Y, Gay CL, Joiner KL, et al. A novel diabetes prevention intervention using a mobile app: a randomized controlled trial with overweight adults at risk. Am J Prev Med. 2015; 49(2): 223-237, doi: 10.1016/j.amepre.2015.01.003, indexed in Pubmed: 26033349.

19. Muralidharan S, Ranjani H, Mohan Anjana R, et al. Engagement and weight loss: results from the mobile health and diabetes trial. Diabetes Technol Ther. 2019;21(9): 507-513, doi: 10.1089/dia.2019.0134, indexed in Pubmed: 31184922.

20. Williams SM, Eleftheriadou A, Alam U, et al.Cardiac autonomic neuropathy in obesity, the metabolic syndrome and prediabetes: a narrative review. Diabetes Ther. 2019; 10(6): 1995-2021, doi: 10.1007/s13300-019-00693-0, indexed in Pubmed: 31552598.

21. Villafaina S, Collado-Mateo D, Fuentes JP, et al. Physical exercise improves heart rate variability in patients with type 2 diabetes: a systematic review. Curr Diab Rep. 2017; 17(11): 110, doi: 10.1007/s11892-017-0941-9, indexed in Pubmed: 28942507.

22. Helleputte S, De Backer T, Lapauw B, et al. The relationship between glycaemic variability and cardiovascular autonomic dysfunction in patients with type 1 diabetes: A systematic review. Diabetes Metab Res Rev. 2020; 36(5): e3301, doi: 10.1002/dmrr.3301, indexed in Pubmed: 32073212.

23. Jaiswal M, McKeon $\mathrm{K}$, Comment $\mathrm{N}$, et al. Association between impaired cardiovascular autonomic function and hypoglycemia in patients with type 1 diabetes. Diabetes Care. 2014;37(9):2616-2621, doi: 10.2337/dc140445, indexed in Pubmed: 24973438. 